Horizons philosophiques

\title{
Un problème d'appropriation : Schleiermacher entre Gadamer et Todorov
}

\section{Brian T. Fitch}

Volume 7, numéro 2, printemps 1997

L’héritage de l’herméneutique

URI : https://id.erudit.org/iderudit/801044ar

DOI : https://doi.org/10.7202/801044ar

Aller au sommaire du numéro

Éditeur(s)

Collège Édouard-Montpetit

ISSN

1181-9227 (imprimé)

1920-2954 (numérique)

Découvrir la revue

Citer cet article

Fitch, B. T. (1997). Un problème d'appropriation : Schleiermacher entre

Gadamer et Todorov. Horizons philosophiques, 7(2), 59-74.

https://doi.org/10.7202/801044ar d'utilisation que vous pouvez consulter en ligne.

https://apropos.erudit.org/fr/usagers/politique-dutilisation/ 


\section{UN PROBLÈME D'APPROPRIATION : SCHLEIERMACHER ENTRE GADAMER ET TODOROV1}

La lecture que propose Gadamer de l'Herméneutique ${ }^{2}$ de Schleiermacher dans la partie dite "historique» de Vérité et méthode a fait couler beaucoup d'encre. L'un des ouvrages les plus récents qui a été consacré à Schleiermacher et dont l'auteur, Christian Berner, avait déjà publié une traduction française de son Herméneutique est allé jusqu'à parler d'une "caricature ${ }^{3}$ de la théorie herméneutique de Schleiermacher. L'interprétation de Gadamer psychologise la méthode exposée par Schleiermacher en la réduisant à un processus de divination afin de s'identifier à l'auteur et en négligeant totalement le rôle de l'interprétation grammaticale. Mais ce qui nous importe ici n'est pas, en premier lieu, le caractère de la déformation que Gadamer a apportée à l'exposition de l'herméneutique de Schleiermacher mais plutôt son attitude devant les textes en question et les raisonnements qu'il a donnés pour se défendre devant ses critiques.

Nous allons donc commencer par résumer rapidement, à l'aide des critiques formulées par Christian Berner, en quoi consiste la partialité de sa lecture de l'Herméneutique. D'abord, il critique le fait que "Gadamer oublie ou tout du moins néglige l'interprétation grammaticale" en faisant remarquer, à juste titre, que «Schleiermacher a toujours insisté sur la complémentarité des deux tâches, psychologique ou technique et grammati-

1. Cet article s'inscrit dans le cadre d'un projet de recherche sur "Le statut et le fonctionnement du métatexte critique dans ses rapports avec le texte littéraire commenté", subventionné par le Conseil de recherche en sciences humaines du Canada.

2. F.D.E. Schleiermacher, Herméneutique, trad. de Christian Berner. Paris : Les Éditions du Cerf/P.U.L., 1987. Signalons l'existence d'une deuxième traduction française de Marianna Simon, avec avant-propos de Jean Starobinski.

Genève : Éditions Labor \& Fides, coll. Lieux théologiques, 1987.

3. La Philosophie de Schleiermacher. Paris : Les Éditions du Cerf, coll. Passages, 1995, p.19. 
cale». Ensuite, il lui reproche de "méconnaître l'ancrage systématique de l'herméneutique dans sa relation avec la dialectique et l'éthique en la rapportant exclusivement à l'esthétique" (19) malgré le fait que "ce qui lui importe [à Schleiermacher] pardessus tout, c'est le contenu cognitif du discours et l'expressif n'a d'importance que dans la mesure où il participe à sa détermination". Finalement, même si l'on accepte la prééminence exclusive accordée à l'interprétation psychologique, «'analyse même de l'interprétation psychologique par Gadamer est fausse dans la mesure où elle s'établit sur l'exclusive connivence du sentiment» de sorte que "la compréhension psychologique est essentiellement compréhension par sentiment» (19-20), tandis qu'en fait «la compréhension psychologique n'est pas chez Schleiermacher une immédiateté du sentiment» mais "une reconstruction hypothétique du discours", car «même l'interprétation psychologique ne fait pas l'économie du caractère constructif de la compréhension, qui suppose non seulement le procédé divinatoire, mais encore le procédé comparatif» (loc. cit.). Ajoutons aux objections de Berner le fait que Gadamer ne fait aucune distinction entre l'interprétation psychologique et l'interprétation technique qu'il réduit à la première.

L'explication de cet état de choses, c'est que Gadamer s'est fondé sur l'édition originelle de l'Herméneutique de 1838 établie par F. Lücke 4 et dont les notes les plus anciennes de Schleiermacher où il était largement question de l'interprétation grammaticale étaient absentes. C'est la nouvelle édition de Heinz Kimmerle ${ }^{5}$ de 1959, faite à l'instigation de Gadamer luimême et publiée un an avant la sortie de Vérité et méthode, qui avait révélé pour la première fois l'importance que Schleiermacher attribuait à l'analyse grammaticale.

Le plus curieux c'est que Gadamer avait déjà connaissance de l'édition Kimmerle dont les manuscrits inédits et originaux, selon les termes de Berner, "semblaient au premier regard

4. Hermeneutik und Kritik mit besonderer Beziehung auf das Neue Testament in Sämmtliche Werke, vol.7. Berlin : Reimer, 1838.

5. Hermeneutik. Nach den Handschriften neu herausgegeben und eingeleitet von Heinz Kimmerle. Heidelberg : Karl Winter, 1959. 
montrer l'extrême importance que Schleiermacher attacha à la structuralité de la langue à travers ce qu'il appelle "l'interprétation grammaticale" qui, quantitativement, est plus développée dans ces notes" (21). Gadamer fait référence dans une note de Vérité et méthode en disant qu'à partir de sa propre impression de l'existence d'une "évolution des pensées herméneutiques de Schleiermacher» qui, écrit-il, "mérite une attention spéciale», Kimmerle réussit "de manière intéressante» à "déterminer l'orientation de l'évolution de Schleiermache» 6 . Cela n'empêche pas pour autant Gadamer de ne pas tenir compte de l'apport de ces nouveaux manuscrits inédits qui va à l'encontre de la lecture de Schleiermacher qu'il propose.

Mais dans la même note en bas de page de son texte, Gadamer avait déjà dit qu'il se fondait sur l'édition originale de Lücke basée sur un manuscrit de 1819 et "surtout à partir de notes de cours de la dernière décennie de Schleiermacher" et il ajoutait ceci : "Ce fait externe montre déjà que la théorie herméneutique que nous connaissons appartient à la dernière période de la pensée de Schleiermacher". - là où on s'attendrait à lire: «la théorie herméneutique que nous connaissions", puisque tel n'est plus le cas au moment de sa rédaction de ces lignes. Et il poursuit: “C'est cette théorie de la maturité qui a exercé une influence historique, surtout par l'entremise de Dilthey. La discussion que nous conduisons ci-haut part de ces textes et cherche à en faire ressortir les tendances essentielles" (205, note 23). Or, comment peut-on prendre pour corpus une édition de textes qu'on sait être incomplète lorsqu'on en a l'édition intégrale à sa disposition? II va sans dire que la situation s'aggrave ici du fait qu'il n'existe aucun manuscrit d'un ouvrage achevé de Schleiermacher intitulé Herméneutique mais seulement une somme de notes de cours, d'ébauches de manuscrits et de discours prononcés magistralement.

Une première réponse se trouve dans la remarque que "[c]'est cette théorie de la maturité qui a exercé une influence historique». Ce serait donc l'héritage historique de l'herméneutique schleiermacherienne dont Gadamer cherche à faire l'ex-

6. Vérité et méthode : les grandes lignes d'une herméneutique philosophique.

Paris : Seuil, édition intégrale : 1996, p.205 note 23. 
position. Mais même ce but-là se trouve hypothéqué par le dernier bout de phrase "surtout par l'entremise de Dilthey". La connaissance de la pensée de Schleiermacher sur laquelle l'héritage en question se fonde se limite à celle, tout à fait insuffisante, qu'en avait Dilthey. Le seul Schleiermacher dont il est question dans l'ouvrage de Gadamer est donc le Schleiermacher de Dilthey, la conception que ce dernier se faisait de son précurseur. N'aurait-il été plus clair de le dire dès le départ?

Gadamer reconnaît l'option qu'il a prise en privilégiant l'interprétation psychologique, lorsqu'il écrit : «Dans ce qui suit, nous laisserons de côté les développements en eux-mêmes très perspicaces que Schleiermacher consacre à l'interprétation grammaticale (grammatisch)" (205). II va jusqu'à avouer qu'«[il] est fort possible - comme le rendent vraisemblable les recherches récentes sur Schleiermacher - que la prédominance de l'interprétation psychologique ne se soit imposée que graduellement dans le développement de la pensée de Schleiermacher" (205). Mais ce n'est que pour revenir à l'argument précité de l'héritage historique : "Quoi qu'il en soit, c'est l'interprétation psychologique qui aura été la plus déterminante pour les théories du XIXe siècle, celles de Savigny, Boeckh, Steinthal et surtout Dilthey«. Plus tard, dans une Postface à Vérité et méthode de 1972, après avoir avoué : «ll se peut que j'aie par trop souligné la tendance de Schleiermacher à l'interprétation psychologique (technique) en face de l'interprétation grammatico-linguistique", il ne fera que revenir au même argument historique : «ll n'en reste pas moins qu'en cela réside son apport le plus personnel, et c'est par suite l'interprétation psychologique qui a fait école $\mathrm{e}^{7}$.

Attardons-nous un moment sur l'inclusion, dans la lignée des précurseurs influencés d'une manière décisive par l'interprétation psychologique schleiermacherienne, d'Auguste Bœckh, étudiant de Schleiermacher et auteur d'une Enzyklopädie und Methodenlehre der philologischen

7. H.-G. Gadamer, L'Art de comprendre. Paris : Aubier, 1982, p.104-5. Cité par Berner in Herméneutique..., p.25 note1. 
Wissenschaft ${ }^{8}$ publiée en 1877 , ainsi que sur la confusion de l'interprétation technique avec l'interprétation psychologique, car il y a un rapport d'interdépendance entre les deux phénomènes qui nous paraît révéler le véritable fondement de la critique de Schleiermacher formulée par Gadamer. En fait, il n'y a pas de trace, à notre avis, de l'interprétation psychologique chez le philologue Boeckh et pour cause - du fait même qu'il était philologue. L'interprétation technique, en revanche, joue, chez lui, un rôle central, complémentaire, comme chez Schleiermacher, de l'interprétation grammaticale qu'il appelle «l'interprétation individuelle», désignation plus à propos car chez lui, comme chez Schleiermacher, il s'agit de cerner la particularité du texte que constitue sa spécificité stylistique. La particularité en question est donc d'ordre langagier et non pas d'ordre psychologique. Contrairement à ce qui semble être l'avis de la plupart des critiques de Gadamer, ce que Gadamer ne peut pas accepter chez Schleiermacher n'est pas uniquement ce qu'il considère être sa psychologisation du texte, mais aussi sa particularisation du texte - ce que Gadamer appellerait sans doute sa singularisation - en se donnant le style pour objet de l'interprétation technique. Cela ressort très clairement du passage suivant de Vérité et méthode :

La compréhension ne devient une tâche particulière que là où cette vie naturelle, dans la communauté de pensée qui est pensée d'une chose commune, se trouve perturbée. Lorsque naissent des malentendus ou lorsque l'expression d'une opinion apparaît incompréhensible, c'est alors seulement que la vie naturelle dans la chose commune se trouve inhibée à tel point que l'opinion en vient à se fixer comme telle, c'est-àdire comme l'opinion de quelqu'un d'autre, d'un toi ou d'un texte.[...] C'est uniquement lorsque tous ces

8. Enzyklopädie und Methodenlehre der philologischen Wissenschaft, E. Bratuscek, éd. Leipzig : Teubner, 1877; 2 e éd. 1886. Réimpression : Darmstadt : Wissenschaftliche Buchgesellschaft, 1977. La deuxième édition de cet ouvrage a été publiée sous une forme abrégée en langue anglaise : August Boeckh, On Interpretation and Criticism, trans., and with an introd. by John Paul Pritchard. Norman : University of Oklahoma Press, 1986, dont des extraits ont été repris par Kurt Mueller-Vollmer dans The Hermeneutics Reader (cf. "Philological Hermeneutics : Philip August Bœckh», p.132-47). 
chemins et ces détours - qui constituent l'art du dialogue, de l'argumentation, de la question et de la réponse, de l'objection et de la réfutation, art qui peut aussi être pratiqué avec un texte dans le dialogue intérieur d'une âme qui cherche la compréhension s'avèrent vains qu'on assiste à un retournement de l'interrogation. Alors seulement, l'effort de la compréhension deviendra attentif à l'individualité du toi et prendra en considération sa singularité 9 .

Voilà la raison pour laquelle Gadamer ne fait aucune distinction entre l'interprétation technique et l'interprétation psychologique schleiermacheriennes car elles sont toutes deux fondées sur la singularité du texte. Mais il importe de faire une distinction entre la singularité psychologique dont s'occupe l'interprétation psychologique, d'une part, et de l'autre, la singularité langagière ou linguistique dont s'occupe l'interprétation technique et qui n'est autre que le style: l'objet de la première est l'esprit de l'auteur, tandis que l'objet de la seconde est le langage du texte. Et du fait d'insister uniquement sur ce dont parle le texte à l'exclusion de la manière dont il en parle qui n'est évidemment pas sans incidence sur son sens, ne serait-ce que par le jeu de la connotation, Gadamer exclut par là même toute considération du style de l'œuvre.

Mais revenons aux arguments avancés par Gadamer. Un autre argument dont il se sert est fondé sur l'originalité respective de l'interprétation psychologique et de l'interprétation grammaticale : «L'herméneutique comprend l'art de l'interprétation grammaticale et psychologique. Mais ce qu'il y a de plus propre à Schleiermacher, c'est l'interprétation psychologique" (206). Faire remarquer que l'interprétation psychologique constitue l'apport le plus original de l'auteur c'est une chose; procéder par la suite à ériger cette forme d'interprétation en système autonome dont l'interprétation grammaticale se trouve purement et simplement éliminée en est une autre... II recourra au même argument en réponse aux objections de Manfred Frank en 1985 : «On ne peut pas cependant à mon sens faire si peu

9. Vérité et méthode. Paris : Seuil, nouv. éd. 1996, p.198-9; Gadamer souligne. 
de cas de l'interprétation psychologique, c'est-à-dire de ce qui est vraiment neuf dans la contribution de Schleiermacher ${ }^{10}$ ".

Dans la même Postface de 1972, Gadamer reconnaît franchement : "[...] mes exposés laissent apparaître une certaine partialité. II en est ainsi à propos de Schleiermacher ${ }^{11}$ ». Mais c'est dans un article de 1968 sur Schleiermacher qu'il explicite le plus clairement la raison de cette «partialité», lorsqu'il écrit :

Dans le cadre systématique de mon essai sur l'herméneutique philosophique, j'ai essentiellement fait appel au travail de Schleiermacher (tout comme de Dilthey) pour établir un contraste critique, lorsque j'ai souligné la signification du mode psychologique d'interprétation comme ce qui est plus propre à Schleiermacher'12.

On voit que Berner a raison lorsqu'il dit que «les vues historiques ont chez Gadamer un statut stratégique», "ses perspectives" étant "fortement orientées par sa propre finalité philosophique» (16-7) 13 . Et pourtant, aux yeux de Gadamer, ce dont il s'occupe, c'est bien l'histoire, car dans sa réponse à Manfred Frank, il s'explique en ces termes : «ll ne s'agissait pas pour moi d'honorer Schleiermacher sous tous ses aspects, mais de le caractériser comme le créateur d'une tradition [Wirkungsgeschichte] [...]. Et cette tradition n'est pas une fiction ${ }^{14}$ ». Le fait d'insister sur une certaine "tradition» est de la plus grande pertinence ici. On connaît le rôle central joué par la tradition dans sa philosophie herméneutique. La tradition est comme un pont qui relie l'horizon de l'interprète dans son présent à lui à l'horizon du texte s'originant dans le passé mais

10. Ibid., p.25. Cité par Berner in Ibid., loc. cit.

11. Ibid., p.25. Cité par Berner in Ibid., p.24 note 1.

12. H.-G.Gadamer, Gesammelte Werke, t.4. Neuere Philosophie, II. Probleme. Gestalten. Tübingen : J.C.B. Mohr, 1987, p.361. Cité par Berner, p.23. Nous soulignons.

13. Berner poursuit en précisant: “ II faut donc rester conscient du fait que la partie historique de Vérité et méthode n'est pas à proprement parler de l'histoire de la philosophie, mais une partie intégrante de l'œuvre elle-même et qu'elle y assure une fonction spécifique et constitutive" (17).

14. L'Art de comprendre, p.26. Cité par Berner, p.25 note1. 
subsistant et se manifestant dans le même présent. Mais la lecture d'une tradition dont la réalité historique ne fait évidemment pas de doute comporte la lecture d'un certain nombre de textes qui jalonnent cette tradition. Et que se passe-t-il lorsque le texte-source, celui qui était à l'origine de la tradition en question, a fait l'objet d'une lecture sinon erronée, clairement partielle, de la part de ses successeurs? Dès lors, cette tradition ne devrait-elle pas être complétée par sa suite qui y a apporté des modifications majeures afin de mieux respecter l'intégrité du système de pensée schleiermacherien rendu nouvellement accessible par la publication de l'édition Kimmerle? Tout se passe ici comme si la lecture d'une tradition importe plus à Gadamer qu'une lecture fidèle du texte sur lequel cette tradition s'est fondée. On est ainsi amené à se demander comment une tradition peut prendre préséance comme objet de l'herméneutique sur le texte des œuvres, lequel est le seul et unique véhicule de la tradition? On est tenté de faire remarquer qu'un tel développement ne peut qu'être facilité par une herméneutique qui privilégie ce dont parle l'œuvre aux dépens du texte qui en assure l'existence, car, pour Gadamer, «la compréhension d'un texte [non-littéraire s'entend] tend à rendre le lecteur accueillant à ce que dit le texte, ce qui signifie précisément sa disparition ${ }^{15}$ ". II est intéressant de constater qu'ici, le texte de Schleiermacher a été occulté précisément par le texte qui en parle, celui de Dilthey.

En fait, Gadamer a choisi de faire comme si ses propres connaissances de l'œuvre de Schleiermacher se limitaient à celles de Dilthey. Et ici, la finalité de sa stratégie entre directement en ligne de compte. On comprendrait sans problème sa façon de faire s'il avait pour but de faire mieux comprendre la lecture que Dilthey a faite de Schleiermacher et de démontrer comment Dilthey avait su profiter de certains aspects de la pensée de Schleiermacher pour élaborer sa propre herméneutique. Mais tel est loin d'être le cas, car Gadamer ne cherche pas à valoriser les idées de Dilthey mais à les critiquer comme étant

15. "Texte et interprétation", in L'Art de comprendre. Écrits II : Herméneutique et champ de l'expérience humaine. Paris : Aubier, coll. Bibliothèque

philosophique, 1991, p.221. 
exemplaires des limitations de la psychologisation et du subjectivisme romantiques. Lire Schleiermacher uniquement à travers son successeur lui permet d'attribuer à sa pensée les mêmes limitations. "La finalité d'une telle stratégie», comme l'observe Berner, "est claire : insister sur la seule interprétation psychologisante et en dévoiler les limites pour critiquer son subjectivisme" (19). Le procès qu'il cherche ainsi à intenter à Schleiermacher est par conséquent nul et non avenu. Le Schleiermacher de Gadamer a tous les attributs d'un homme de paille créé pour les besoins de la cause.

Nous avons vu que Gadamer dit - après coup, vingt-cinq ans après la parution de Vérité et méthode - avoir voulu "caractériser Schleiermacher comme créateur d'une tradition". II aurait pu dire qu'il étudiait l'historique de la réception de Schleiermacher. Mais qui dit «tradition» dit «appropriation». Car qu'est-ce qu'une tradition, dans le présent contexte, sinon le résultat d'une réception arrêtée, faite une fois pour toutes, jamais plus mise en question ni renouvelée? Ce que fait Gadamer, c'est cautionner une certaine appropriation historique de l'œuvre de Schleiermacher, qu'il sait, pourtant, être dépassée du fait de la publication de manuscrits inédits. II la cautionne par le fait même de l'assumer, de la faire sienne, en racontant l'histoire de la naissance d'une tradition dans le domaine de l'histoire des idées. II s'agit donc de l'appropriation d'une appropriation. Il est pour le moins ironique que celui qui a été largement responsable de la réhabilitation du concept d'«appropriation», qu'il a rebaptisé «application», - concept totalement absent de la pensée de Schleiermacher - ne se sert jamais de son propre concept en parlant de la filiation historique entre Schleiermacher et Dilthey, ce qui aurait été la seule façon d'éviter tout malentendu quant au statut de sa propre lecture de Schleiermacher.

Passons maintenant à une deuxième appropriation de la pensée schleiermacherienne et qui, tout en étant aussi partiale et partielle que celle de Gadamer, se pose curieusement en opposition symétrique à la sienne. II s'agit de la lecture de son 
Herméneutique que propose Tzvetan Todorov dans son ouvrage Symbolisme et interprétation ${ }^{16}$. Plutôt que de privilégier l'interprétation psychologique schleiermacherienne, Todorov la passe sous silence en insistant uniquement sur l'interprétation grammaticale et la première forme de l'interprétation psychologique qu'était l'interprétation technique. Curieusement cette lecture constitue l'exacte contrepartie de l'interprétation de Schleiermacher qui figure dans Vérité et méthode puisque là où Gadamer psychologise sa méthode herméneutique, Todorov la textualise. L'intérêt principal de la lecture de Todorov est de permettre de juger du degré de compatibilité qui existe entre les textes de Schleiermacher et la méthodologie de la poétique moderne. L'essentiel de la stratégie interprétative que révèle cette lecture incontestablement innovatrice - et pour cause, comme nous le verrons réside dans le fait de privilégier à tout prix le concept du texte comme point de départ et comme point d'aboutissement de la démarche herméneutique schleiermacherienne et ce faisant, d'absoudre son auteur de tout reproche de psychologisme. On pourrait fort bien voir la finalité d'un tel travail dans une sorte de réhabilitation de Schleiermacher à la lumière de la poétique moderne.

L'originalité de L'Herméneutique se trouve, pour Todorov, dans l'importance qu'elle attribue à l'interprétation formelle intratextuelle, ce qu'il appelle "l'interprétation "structurelle"», qu'il estime avoir été abandonnée par les herméneutes depuis Spinoza. Afin de mettre en valeur la place prépondérante du texte chez Schleiermacher, Todorov traduit l'ensemble des concepts et des stratégies interprétatives à l'œuvre en termes textuels. L'exemple le plus frappant en est la définition qu'il donne de l'auteur comme étant "un contexte syntagmatique». Une telle définition - si elle n'est pas tout à fait erronée dans la mesure où l'auteur, chez Schleiermacher, est bien un être de caractère langagier - simplifie à l'extrême la forme sous laquelle se manifeste l'auteur dans ses textes.

16. Paris : Seuil, 1978. 
C'est que Todorov choisit de perdre de vue entièrement l'un des traits fondamentaux de son herméneutique qu'est l'indissociabilité du langage et de la pensée. Le langage est la manifestation tangible de celui qui s'en sert. II est d'autant plus ironique que Todorov ne paraît apprécier ni la priorité, souvent explicitée, que Schleiermacher accorde au discours oral ni la situation dialogique dont il est inséparable, lui qui, trois ans après la publication de Symbolisme et interprétation, devait consacrer un ouvrage au principe dialogique de Mikhaïl Bakhtine ${ }^{17}$ avant d'épouser lui-même le concept d' «une critique dialogique ${ }^{18}$ ». Comme le fait remarquer à juste titre Hans Robert Jauss, "Le fait que Schleiermacher passe de la compréhension des textes à la compréhension du discours en général veut dire qu'on a besoin d'une herméneutique de la dialogicité, herméneutique qui, même aujourd'hui, n'est toujours pas disponible ${ }^{19}$ ". Loin d'être une présence muette, sans voix, le texte schleiermacherien devient, pour chaque lecteur, un interlocuteur dont il s'agit d'interpréter la parole qu'incarne le texte, de même qu'il faut interpréter la parole de celui avec qui on s'entretient. L'altérité du langage du texte n'est ni celle d'une matière langagière inerte ni l'abstraction d'un code correspondant au système linguistique, comme le voudrait le structuralisme, mais celle de l'autre dont la pensée est véhiculée par le discours du texte.

Une telle façon de présenter la pensée de Schleiermacher n'est ni plus ni moins partielle et partiale que celle de Gadamer. Ici, aussi, il s'agit d'une interprétation des textes qui ne rend absolument pas compte de la totalité du système de pensée qu'ils représentent et par là même tend à revêtir tous les attributs d'une caricature. Le mot «appropriation" vient, d'ailleurs, se présenter encore plus vite à l'esprit dans le cas de Todorov - en termes polémiques, on serait tenté de parler d'une «récupération» de Schleiermacher. Ajoutons que vu les présup-

17. Mikhail Bakhtine : le principe dialogique suivi d'écrits du cercle de Bakhtine.

Paris : Seuil, 1981.

18. "Une Critique dialogique?" in Critique de la critique. Paris : Seuil, 1984, p.17993.

19. Question and Answer : Forms of Dialogic Understanding. Minneapolis : University of Minnesota Press, 1989, p.214. Nous traduisons de l'anglais. 
posés théoriques du poéticien, auteur de Symbolisme et interprétation, l'appropriation qui y a été faite de l'œuvre de son précurseur n'aurait sans doute pas pu se présenter autrement. Dans le contexte des études littéraires françaises des années 70, la lecture de Todorov s'imposait en quelque sorte. Comme l'a bien dit Gadamer, toujours et par la force des choses, "on comprend autrement» 20 , selon la situation historique de celui qui comprend et même selon le moment où s'effectue l'acte de compréhension.

Examinons de plus près ce qui est à l'œuvre dans le processus de l'appropriation chez Gadamer et chez Todorov. Tous deux procèdent à une certaine contextualisation de l'œuvre de Schleiermacher. Dans le cas de Gadamer, il s'agit d'une contextualisation diachronique où le contexte en question est constitué par l'évolution historique de sa réception; dans le cas de Todorov, il s'agit d'une contextualisation synchronique de caractère a-temporel où le contexte est constitué par le champ de concepts faisant partie d'une certaine théorie de la littérature, la poétique. Dès que quelque chose se trouve contextualisé, ce que la chose en question partage avec son contexte se trouve valorisé et ce qui l'en différencie se trouve relégué à l'arrière-plan, telle l'analyse grammaticale schleiermacherienne chez Gadamer et l'analyse psychologique schleiermacherienne chez Todorov.

Une deuxième manière de rendre compte du phénomène de l'appropriation auquel nous assistons ici serait de dire que le processus de la contextualisation est précédé par celui d'une décontextualisation - ici, décontextualisation de tel ou tel type d'analyse schleiermacherien - et que ce sont les éléments décontextualisés de l'œuvre commentée qui seront par la suite recontextualisés. Dès lors, le premier contexte d'où ces éléments auront été sortis serait l'ensemble de la pensée de Schleiermacher contenue dans l'ensemble des textes publiés dans l'édition de son Herméneutique. 
Ce que cela revient à dire, dans la terminologie gadamérienne, c'est que chez lui, comme chez Todorov, son propre horizon a prévalu au détriment de l'horizon de l'œuvre commentée. Pour passer de la description à la prescription, il faudrait, en revanche, que le travail de recontextualisation qui caractérise nécessairement tout travail d'appropriation ne soit pas précédé par la décontextualisation de certains éléments constitutifs de l'œuvre. Ce qu'il convient de contextualiser est l'œuvre dans son ensemble. Mais notons que cette contextualisation sera toujours, en fait, une recontextualisation, mais le contexte d'origine dont il s'agira ne sera plus celui constitué par l'ensemble du texte mais celui à l'intérieur duquel le texte avait pris sa place, lors de sa rédaction : contexte intellectuel, sociologique, etc. À ce dernier contexte sera substitué le nouveau contexte constitué par l'horizon issu de la suite de la fusion d'horizons, celui de l'œuvre et celui du commentateur. II va de soi que la lecture de l'œuvre qui en sera le résultat aura le statut d'une interprétation. Celle-ci sera tout aussi partiale que celles proposées par Gadamer et Todorov, mais, en revanche, elle ne sera plus partielle.

Gadamer et, à son tour, Paul Ricœur ont bien démontré qu'il ne saurait y avoir de lecture et donc d'interprétation de texte sans appropriation. Et ces deux interprétations de Schleiermacher en témoignent d'une manière exemplaire. Chacun de ces deux lecteurs ne voit dans les textes de Schleiermacher que ce qu'il veut bien y voir. Sa compréhension est orientée nécessairement par ses propres préoccupations théoriques et par la nature de la synthèse de l'évolution de l'herméneutique qu'il cherche à élaborer. Ce qui est difficilement compréhensible pour le lecteur de Gadamer et de Todorov, c'est que, malgré que leur propos soit le processus herméneutique de l'interprétation des textes, ils n'aient pas su prendre conscience du fait de se trouver eux-mêmes pris dans ce même processus et sujets à ses contraintes et à ses limitations dont ils reconnaissent bien, par ailleurs, l'existence. Prétendre interpréter les écrits de l'un des maîtres de l'herméneutique sans manifester aucune conscience méthodologique du statut de son 
propre métadiscours est, à notre sens, le comble de l'ironie. Encore plus ironique est la constatation que c'est précisément une véritable maîtrise du système d'interprétation proposé par Schleiermacher qui leur aurait permis d'éviter des erreurs d'interprétation si flagrantes. Deux exemples : pour bien comprendre et interpréter les pages consacrées à Schleiermacher par Gadamer, nous devons les situer par rapport à l'ensemble de la synthèse qu'il propose de l'historique de l'évolution de la pensée herméneutique selon la méthodologie des contextualisations de plus en plus larges proposée par Schleiermacher luimême. Le même procédé de méthodologie aurait permis à Gadamer de tenir compte de "l'ancrage systématique de l'herméneutique dans sa relation avec la dialectique et l'éthique" (loc. cit.), dont parle Berner, et d'éviter de la rapporter "exclusivement à l'esthétique". Mais le fait est qu'à cause même de leur stratégie interprétative défectueuse, la méthode de Schleiermacher en tant que telle, dans son intégralité, ne leur était pas accessible.

Pour conclure, revenons à Schleiermacher lui-même et à son héritage dans lequel les interprétations de Gadamer et de Todorov sont venues prendre leur place. La clé de voûte de son système se trouve, comme nous l'avons dit, dans l'indissociabilité du langage et de la pensée. Les deux grandes tendances chez ses successeurs choisissent de privilégier soit le langage (tel Todorov) soit la pensée (tel Gadamer). C'est Ricœur qui a fait bien ressortir l'opposition entre ces deux interprétations qui se centrent, d'une part, sur l'interprétation technique et grammaticale et de l'autre, sur l'interprétation psychologique :

L'interprétation technique n'est pas vraiment ce qu'il appelle 'l'interprétation psychologique' dans les textes de la dernière période. Car la singularité d'une œuvre peut être comprise de deux manières différentes. On peut dire que ce qui est singulier est l'idée qui informe l'œuvre, ce qu'il appelle en termes plus détaillés «la forme interne d'une œuvre». [...] L'individualité peut 
donc être conçue comme un réseau systématique dans lequel les parties sont subordonnées à l'ensemble. [...] Mais on peut aussi dire que l'individualité d'une œuvre provient du complexe psychologique dont émane cette idée 21 .

Et Ricœur de poursuivre : «Schleiermacher n'est jamais arrivé à départager clairement ces deux orientations possibles de l'interprétation technique : vers l'idée qui informe l'œuvre ou vers l'auteur considéré comme un être psychologique». Ses successeurs, en revanche, n'ont pas éprouvé la même difficulté. C'est précisément au prix de séparer l'inséparable, le langage et la pensée, - qui, tout comme le signifiant et le signifié saussuriens, sont le recto et le verso de la même feuille de papier et qui se rejoignent dans le concept de «style»22 chez Schleiermacher - qu'ils arrivent aux lectures partielles et partiales telles que celles que nous venons d'examiner.

II nous paraît significatif que le texte de Schleiermacher a pu donner lieu à deux lectures qui s'opposent totalement l'une à l'autre. La première, celle de Gadamer, trouvera son point d'aboutissement dans l'œuvre critique d'un Georges Poulet qui conçoit le rapport entre le lecteur-interprète et le texte comme la rencontre de deux consciences : «L'acte de lire [...]", écrit-il, «implique la coïncidence de deux consciences : celle du lecteur et celle d'un auteur ${ }^{23}$ ". La deuxième, celle de Todorov, dans les travaux des poéticiens. Comme Peter Szondi24 et, à sa suite, Manfred Frank25 l'ont souligné à juste titre, bien des concepts

21. "Schleiermacher's Hermeneutics", The Monist, vol.LX, no.2, April 1977, p.188. Nous traduisons de l'anglais.

22. "Between these two directions - pointing to the text and pointing to the author - hermeneutics hesitates. And yet, Schleiermacher did, in the idea of style, glimpse their profound unity." (Ricœur, art. cité, loc. cit.; Ricœur souligne).

23. La Conscience critique. Paris : Corti, 1971, p.9.

24. Introduction à l'herméneutique littéraire: de Chladenius à Schleiermacher. Paris : Les Éditions du Cerf, 1989, et "Schleiermacher's Hermeneutics Today" in On Textual Understanding and Other Essays. Minneapolis : University of Minnesota Press, 1986, p.95-113.

25. Voir "L'Herméneutique de Schleiermacher. Relecture autour du débat Herméneutique- Néostructuralisme", Revue internationale de philosophie, 38 e année, no.151, 1984, p.348-71, et Qu'est- ce que le Néo-structuralisme : de Saussure et Lévi-Strauss et Lacan. Paris : Éditions du Cerf, coll. Passages, 1989. Traduit de l'allemand par Christian Berner : Was ist Neostrukturalismus. Frankfurt-am- Main : Surkamp Verlag, 1984 
opératoires dans les débats contemporains en théorie littéraire se trouvent compris dans le système de Schleiermacher. Mais il ne s'agit pas de sortir de son contexte, c'est-à-dire de l'ensemble de sa pensée herméneutique, tel ou tel concept, ni telle ou telle démarche méthodologique sans aucun égard pour leur intercomplémentarité par rapport aux autres concepts ou aux autres démarches, intercomplémentarité sur laquelle Schleiermacher ne cesse d'insister sans, malheureusement, être entendu. En dernière analyse, l'essentiel, les principes de base, des deux grandes tendances en théorie littéraire, l'herméneutique et la poétique, coexistent chez Schleiermacher. Le sort de sa pensée quant à l'évolution ultérieure des études littéraires a été de se trouver scindée en deux.

\author{
Brian T. Fitch \\ Département d'études françaises \\ Université de Toronto
}

